\title{
Caesarean Section in African Setting: Current Situation, Problematic and Qualitative Approaches at Laquintinie Hospital (Douala, Cameroon)
}

\author{
Essome Henri ${ }^{1,2}$, Mve Koh Valere ${ }^{3,4}$, Engbang Jean Paul ${ }^{1,2}$, Boten Merlin', Essiben Felix ${ }^{4,7}$, \\ Tocki Toutou Grâce1, Foumane Pascal4,5, Mboudou Emile Telesphore ${ }^{4,6}$ \\ ${ }^{1}$ Laquintinie Hospital of Douala, Douala, Cameroon \\ ${ }^{2}$ Faculty of Medicine and Pharmaceutical Sciences, University of Douala, Douala, Cameroon \\ ${ }^{3}$ University Teaching Hospital Yaoundé, Yaoundé, Cameroon \\ ${ }^{4}$ Faculty of Medicine and Biological Sciences of Yaoundé, University of Yaoundé 1, Yaoundé, Cameroon \\ ${ }^{5}$ Gynaeco-Obstetrics and Paediatric Hospital of Yaoundé, Yaoundé, Cameroon \\ ${ }^{6}$ Gynaeco-Obstetric and Paediatric Hospital of Douala, Douala, Cameroon \\ ${ }^{7}$ Central Hospital of Yaoundé, Yaoundé, Cameroon \\ Email: *essometocky@yahoo.com
}

How to cite this paper: Henri, E., Valere, M.K., Paul, E.J., Merlin, B., Felix, E., Grâce, T.T., Pascal, F. and Telesphore, M.E. (2019) Caesarean Section in African Setting: Current Situation, Problematic and Qualitative Approaches at Laquintinie Hospital (Douala, Cameroon). Open Journal of Obstetrics and Gynecology, 9, 1392-1406. https://doi.org/10.4236/ojog.2019.910135

Received: September 6, 2019

Accepted: October 22, 2019

Published: October 25, 2019

Copyright $\odot 2019$ by author(s) and Scientific Research Publishing Inc. This work is licensed under the Creative Commons Attribution International License (CC BY 4.0).

http://creativecommons.org/licenses/by/4.0/

\begin{abstract}
Background: Quality control of care aims to reduce or eliminate unnecessary care and to improve the quality of those who are useful both in their indication and in their implementation. Objective: We conducted this study to assess the rate of caesarean section, the rate of irrelevant indications, materno-foetal morbidity and mortality, biases in the management in order to suggest corrective approaches. Methods: It was a cross-sectional study conducted in the gynaeco-obstetrics department of the Laquintinie Hospital of Douala over a period of 4 months from January 1 to April 30, 2017. We included all pregnant women who had a caesarean section and who gave consent to our study as well as new-borns from these caesareans. We excluded caesarean deliveries from other health structures and referred to Laquintinie Hospital due to morbid operative follow-up. The variables collected were grouped under 3 main headings: socio-demographic data, clinical data and post-operative follow-up. Results: A total of 281 caesarean sections were performed out of a total of 967 deliveries; a caesarean section rate of $29.06 \%$ in 4 months. After data mining, 250 caesarean sections were included in the study because 31 cases of caesarean deliveries were unusable. Referred pregnancies accounted for $46.8 \%$ of the total population and the most common reason for referral was stationary labour (23\% of cases). All caesareans were
\end{abstract}


performed by the gynaeco-obstetricians. Women who had caesarean deliveries were informed by the operator of the surgical procedure in $28.4 \%$ of cases and $27.6 \%$ were notified of the indication for surgery. The operative kit was present in $98 \%$ of cases. The operating room was available in $93 \%$ of cases. Caesareans were performed mostly in an emergency context (91.2\%) with a median turnaround time of 214 minutes ( $3 \mathrm{~h} 56$ mins). Mechanical dystocia was the major indication in our series $(21.2 \%)$ and 29 indications were irrelevant (11.6\%). Intraoperative complications occurred in $3.2 \%$ of cases. Overall maternal mortality (per- and post-operative) was $0.8 \%$ ( 2 cases). We recorded 15 neonatal deaths out of which 8 were still births. Regarding the postoperative period, $78 \%$ of the operated patients did not have a good immediate postoperative monitoring. The post-operative protocol was not respected in $17 \%$ of cases. Postoperative complications occurred in $21.6 \%$ of patients with first cause being infections ( $10.8 \%$ with $5.6 \%$ being parietal suppurations). Conclusion: The frequency of Caesarean sections at Laquintinie Hospital is above the World Health Organization's recommendations of 5\% - 15\%. There is a very big delay in the execution time of emergency caesareans, far above the international standards despite the quasi-availability of operating kits and the operating theatre. The state of the premises reveals a sub-workforce creating work overload and therefore a demotivation of the staff leading to insufficient communication between the operator and operated, a lack of postoperative follow-up and significant neonatal morbidity and mortality. Hence the need to initiate a staff satisfaction survey.

\section{Keywords}

Caesarean Section, Practical, Laquintinie, Cameroon

\section{Introduction}

To address maternal mortality and neonatal complications related to vaginal delivery, caesarean section has become an increasingly popular surgical procedure around the world. $24.1 \%$ of births by caesarean section in the United States; $14.06 \%$ of births in Djibo, Burkina Faso in 2014; $18.64 \%$ and $23.73 \%$ respectively at the central hospital and the General Hospital Yaoundein Cameroon in 2012. These figures thus appear far from the $5 \%-15 \%$ birth rate recommended by WHO [1] [2].

Nevertheless, this intervention is associated with a risk of materno-foetal morbidity and mortality. In industrialized countries, maternal mortality rate related to caesarean section from $0.07 \%$ to $0.34 \%$ has been reported in the literature [3]. In Africa, particularly in Mali and Cameroon, morbidity rates of 22.7\% and $16.95 \%$ respectively are reported [1] [4].

The outbreak of caesareans that the world knows in recent decades challenges us on the problem of this intervention and the evaluation of its practice.

The evaluation of medical practices is a set of processes aimed at helping the 
health professional to make the best diagnostic and therapeutic choice for the patient and the community. This evaluation appreciates the therapeutic results, the sequalae of the treatment as well as the satisfaction of the patient at the end of the hospital stay.

This quality control of care aims to reduce or eliminate unnecessary care and improve the quality of necessary care both in its indication and implementation.

Quality caesarean section is defined as an intervention that benefits all pregnant women who really need it with minimal risk for mother and child and has an affordable cost for the health system.

The caesarean section rate is a good indicator of the quality process of obstetric care. Too low, it reflects poor coverage in obstetric care; too high, it is suggestive of a deleterious practice with questionable indications. The WHO standard is $5 \%$ to $15 \%$ [1] [5].

The maternal mortality rate in Cameroon is 782 deaths per 100,000 live births; this rate is indicative of a lack of organization or low coverage of obstetric care and the quality of these. In Cameroon a woman dies every 2 hours giving birth, that is 12 each day [6]. In 2015, the same report estimated neonatal mortality at 596 deaths per 100,000 births in Cameroon [7].

Many countries around the world in general, and in Africa in particular, have realized the need to evaluate the quality of care to improve it. Regarding the practice of caesarean section, there are studies done in Senegal in 1996, in Burkina Faso in 2005 [8] [9]. In Cameroon, few studies in the sense of assessing the quality of care in general and obstetrics, in particular, are conducted. This scarcity of study and the high morbidity and mortality in obstetrics in our country have led us to look at a decisive element of maternal morbidity and mortality, notably caesarean section in order to evaluate its practice at Laquintinie Hospital in Douala which falls under the central level of Cameroon's health pyramid.

\section{Methods}

\subsection{Type of Study}

This was a cross-sectional descriptive study that took place from January 1, 2017 to April 30, 2017 (i.e., 4 months) in the post-operative section at the maternity of the laquintinie hospital in Douala, Cameroon.

The Laquintinie Hospital is a university-hospital setting that records 2900 births a year, of which nearly one-third (950) by caesarean section for 06 gynaecologists. The obstetrics and gynaecology department has a capacity of 80 beds and a fill rate of $80 \%$. The shifts at the maternity clinic are attended for a period of 24 hours (8:00 to 8:00) for the gynaecologist assisted by a rotating team of paramedics by sequential period (the morning from $8 \mathrm{am}$ to $5 \mathrm{pm}$ and the night from $5 \mathrm{pm}$ to $8 \mathrm{am}$ ) and consisting of a nurse anaesthetist, two nurses in the operating room, 3 nurses midwives and 3 general nurses, the posto-perative section has a capacity of 36 beds for two nurses during day and night shifts. According to an empirical ratio of 1 paramedical guard for 8 beds, the ratio of operative 
follow-up in the department of obstetrics and gynaecology of the laquintinie hospital is 1 nurse for 18 beds. The work overload and difficulties faced are therefore normal. The provision of running water is subject to multiple cuts and refuelling is not always non-contaminating. The sterilization chain is defective and prone to multiple breakdowns.

\subsection{Study Population}

We recruited women who underwent caesarean deliveries at Laquintinie hospital in Douala who consented to our study, as well as new-borns from these caesareans. Sampling was consecutive, non-exhaustive. We excluded caesarean deliveries from other health structures and referred to Laquintinie Hospital due to morbid operative follow-up.

\subsection{Materials}

The information was collected by direct interrogation using a pre-tested form; but also, by examining their medical records and operating room registers. An informed consent form validated by the ethics committee of the University of Douala was read beforehand to the members with their signature. The variables selected for the study were socio-demographic (age, marital status, occupation, residence and religion) the surgical indications, the conditions of management, the duration of the interventions, the post-operative follow-up and complications, the maternal and neonatal deaths. Caesareans were divided into 5 categories:

Absolute emergencies: that is, the life of the mother and/or the fet we is in immediate danger if the caesarean section is not performed (uterine rupture or uterine rupture syndrome, placenta praevia covering haemorrhage, cord prolapse).

Mandatory: absence of intervention exposing to maternal death and/or serious maternal sequelae (cephalo-pelvic disproportion, vicious presentation, narrowed pelvis).

Absolute need: corresponds to pathologies associated with pregnancy or abnormalities of delivery (hypertensive pathology, dynamic dystocia).

Caesarean section of caution: corresponds to situations for which intervention is not necessary; vaginal delivery is possible, but the surgical procedure is considered useful to improve the functional prognosis of the mother as well as a well-being to the new-born (fresh single uterine scar).

Non-relevant indications: dynamic dystocia without previous correction, limited pelvis without test of labour.

The analysis of this data was carried out with SPSS software (Statistical Package for Social Sciences) version 17 and Microsoft Excel 2010.

\section{Results}

During our 4-month study, we recorded 967 deliveries (all routes included) in- 
cluding 281 caesarean sections (29.06\%). However, only 250 (documented) caesarean sections were included (31 excluded due to lack of exploitable information). The adherents were mostly between 20 - 35 years of age, unemployed, married, living in disadvantaged neighbour-hoods and of Christian religion (Table 1) there were 228 (91.2\%) caesareans performed in an emergency setting. About half of these emergency caesarean sections were referred cases: 117 cases (46.8\%). The predominant reason for the referral was stationary labour: $23.9 \%$ (28 cases) (Table 2) and $11.6 \%$ (29 cases) of the indications were irrelevant (Table 3). The major surgical indication of our series was mechanical dystocia 53 cases $(21.2 \%)$ (Table 3 ).

Of our series of 250 caesareans, $28.4 \%$ were informed of the operative procedure (71 cases) and $27.1 \%$ (69 cases) were able to respond on the indication of their caesarean section (Table 4).

Of the 228 emergency Caesarean sections performed, 95.6\% (218 cases) were performed greater than 1 hour after decision making (Figure 1). The median turnaround time was 214 minutes (approximately $3 \mathrm{~h} 56$ minutes), with a minimum of 20 minutes and a maximum of 2104 minutes (approximately 35 hours).

The duration of the intervention was not specified in most files. However, out of 143 cases in this variable, $73.42 \%$ (105 cases) had an intervention duration of less than 60 minutes (Table 4).

All these caesareans were performed by gynaecologists-obstetricians.

Table 1. Distribution of the pregnant women with respect to socio-demographic characteristics.

\begin{tabular}{|c|c|c|c|}
\hline Variables & Categories & Frequency $(\mathrm{N}=250)$ & Percentage (\%) \\
\hline \multirow{3}{*}{ Age } & $\leq 19$ years & 10 & 4 \\
\hline & 20 - 35 years & 190 & 76 \\
\hline & $>35$ years & 50 & 20 \\
\hline \multirow{5}{*}{ Occupation } & Unemployed & 83 & 33 \\
\hline & Self-employed & 65 & 26 \\
\hline & Pupil/student & 55 & 22 \\
\hline & Private sector & 32 & 13 \\
\hline & Public sector & 15 & 6 \\
\hline \multirow{2}{*}{ Marital status } & Married & 157 & 62.8 \\
\hline & Single & 93 & 37.2 \\
\hline \multirow{3}{*}{ Residence } & Residential neighbour & 92 & 37 \\
\hline & hood & 158 & 63 \\
\hline & Un-favoured neighbourhood & & \\
\hline \multirow{2}{*}{ Religion } & Christian & 235 & 94 \\
\hline & Muslim & 15 & 6 \\
\hline
\end{tabular}


Table 2. Distribution with respect to reason for admission of the referred pregnant women.

\begin{tabular}{ccc}
\hline Reason for admission & Frequency $(\mathrm{N}=117)$ & Percentage (\%) \\
\hline Stationary labour & 28 & 23.9 \\
Severe pre-eclampsia & 15 & 12.8 \\
AFD $^{1}$ & 14 & 11.9 \\
PRM $^{2}$ & 12 & 10.25 \\
CPD $^{3}$ & 11 & 9.40 \\
LPP $^{4}$ similar to UC & 10 & 8.54 \\
Eclampsia & 8 & 6.83 \\
Cord prolapse & 6 & 5.12 \\
Uterine scar & 5 & 4.27 \\
Multi-scared uterus & 2 & 1.7 \\
Bleeding & 2 & 1.7 \\
Post-term & 2 & 1.7 \\
Abnormal presentation & 2 & 1.7 \\
\hline
\end{tabular}

$\mathrm{AFD}^{1}$ : Acute foetal distress, $\mathrm{PRM}^{2}$ : Prolonged rupture of membranes, $\mathrm{CPD}^{3}$ : Cephalo-pelvic disproportion, $\mathrm{UC}^{4}$ : Uterine contractions, LPP ${ }^{5}$ : Lombo-pelvic pain.

Table 3. Distribution of types of caesarean indications.

\begin{tabular}{|c|c|c|}
\hline Type of caesarean & Frequency $(\mathrm{N}=250)$ & Percentage (\%) \\
\hline \multicolumn{3}{|l|}{ 1) Absolute emergencies } \\
\hline$-\mathrm{AFD}^{1}$ & 35 & 14 \\
\hline$-\quad \mathrm{PPCH}^{2}$ & 14 & 5.6 \\
\hline - Cord prolapse & 7 & 2.8 \\
\hline - Pre-rupture syndrome & 6 & 2.4 \\
\hline \multicolumn{3}{|l|}{ 2) Mandatory } \\
\hline - Mechanical distocia & 53 & 21.2 \\
\hline - Repeated indication & 23 & 9.2 \\
\hline \multicolumn{3}{|l|}{ 3) Absolute need } \\
\hline - Severe pre-eclampsia & 15 & 6 \\
\hline - Eclampsia & 11 & 4.4 \\
\hline - Stationnary Labou & 12 & 4.8 \\
\hline - $\mathrm{RPH}^{3}$ & 4 & 1.6 \\
\hline \multicolumn{3}{|l|}{ 4) Caesarean of caution } \\
\hline - Fresh single uterine scar & 41 & 16.4 \\
\hline \multicolumn{3}{|l|}{ 5) Non-relevant indications } \\
\hline - $\quad \mathrm{PRM}^{4}$ & 18 & 7.2 \\
\hline - Suspicion of macrosomia & 6 & 2.4 \\
\hline - Post term & 3 & 1.2 \\
\hline - Chorio-amnionitis & 2 & 0.8 \\
\hline
\end{tabular}

$\mathrm{AFD}^{1}$ : Acute foetal distress, $\mathrm{PPCH}^{2}$ : Placenta praevia covering haemorrhage, $\mathrm{RPH}^{3}$ : Retro-placental haemorrhage, $\mathrm{PRM}^{4}$ : Prolonged rupture of membranes. 
Table 4. Conditions of care.

\begin{tabular}{|c|c|c|}
\hline Variables & Frequency $(\mathrm{N}=250)$ & Percentage (\%) \\
\hline Availability of kits & 245 & 98 \\
\hline Availability of theatre & 333 & 93 \\
\hline Informed of the procedure & 71 & 28.4 \\
\hline Informed of the indication & 69 & 27.6 \\
\hline Emergency caesarean & 228 & 91.2 \\
\hline Planned caesarean & 22 & 8.8 \\
\hline General anaesthesia & 37 & 15 \\
\hline Spinal anaesthesia & 213 & 85 \\
\hline \multicolumn{3}{|l|}{ Duration of intervention } \\
\hline$<30$ mins & 25 & 17.48 \\
\hline 30 mins - 1 hour & 105 & 73.42 \\
\hline 1 hour -2 hours & 12 & 8.39 \\
\hline$>2$ hours & 1 & 0.70 \\
\hline \multicolumn{3}{|l|}{ Post-op monitoring } \\
\hline \multicolumn{3}{|l|}{ Every 30 mins for 2 hours } \\
\hline \multicolumn{3}{|l|}{ First hours } \\
\hline YES & 55 & 22 \\
\hline NO & 195 & 78 \\
\hline \multicolumn{3}{|l|}{ Respect of the surgical protocol } \\
\hline YES & 205 & 82 \\
\hline $\mathrm{NO}$ & 45 & 18 \\
\hline
\end{tabular}

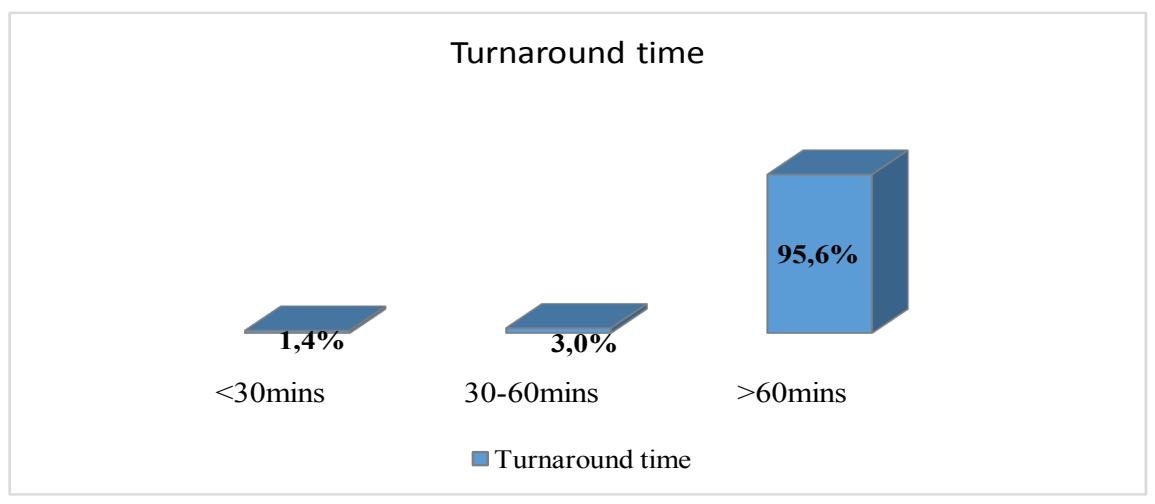

Figure 1. Turnaround time for the surgical action.

Two types of anaesthesia were performed at Laquintinie Hospital maternity ward: general anaesthesia and spinal anaesthesia with a predominance of spinal anaesthesia (85\% of cases) (Table 4 ).

During the study period, we recorded intraoperative haemorrhages by a percentage three times higher (1.2\%) (Table 5) than intraoperative maternal death due to anaesthetic causes ( $0.4 \%$ of caesarean section) (Table 6$)$. 
Table 5. Per-operative complications.

\begin{tabular}{lcc}
\hline \multicolumn{1}{c}{ Variables } & Frequency $(\mathrm{N}=250)$ & Percentage (\%) \\
\hline 1) Complications due to anaesthesia & 1 & 0.4 \\
\hline - Vasoplegia & 1 & 0.4 \\
- Cardio-respiratory arrest & 3 & 1.2 \\
2) Per-operative haemorrhage & 1 & 0.4 \\
3) Bladder injury & 1 & 0.4 \\
4) Hematoma of the large ligament & & \\
\hline
\end{tabular}

Table 6. Materno-fotal mortality.

\begin{tabular}{ccc}
\hline Variables & Frequency $(\mathrm{N}=250)$ & Percentage (\%) \\
\hline Maternal Death & 1 & 0,4 \\
\hline Still birth & 8 & 3,2 \\
Transfers to neonatology & 26 & 10,4 \\
Deaths in neonatology & 7 & 2,8 \\
Total neonatal deaths & 15 & 6 \\
Live new-borns who left the hospital & 235 & 94 \\
\hline
\end{tabular}

We recorded 8 stillbirths (3.2\%), 216 new-borns (86.4\%) had a satisfactory APGAR score at the first minute and 26 neonatal transfers (10.4\%) for apparent death. At discharge from the hospital, 235 new-borns (94\%) were alive compared to 7 neonatal deaths (2.8\%). Giving a total of 15 neonatal deaths $(6 \%)$.

Only 55 (22\%) patients were monitored every 30 minutes for the first 2 hours.

The postoperative protocol was not respected in $18 \%$ of cases.

The follow-up was enamelled with 54 cases $(21.6 \%)$ of complications with a predominance of infectious complications: 27 cases (10.8\% of the general study population) (Figure 2).

\section{The Limits of Our Study}

This study was a punctual and essentially descriptive look at the practice of caesarean section at the laquintinie hospital. We did not do statistical analyses with confusion checks by logistic regressions in this study. For this purpose, we plan to carry out further analytical studies in an exclusive qualitative approach. We discussed our results according to the variables retained while discriminating those which did not seem to us reliable; because poor record keeping and inconsistent reporting of some data prejudged bias in analysis and relevance.

\section{Discussion}

The optimal age range for human procreation is $20-30$. The age of caesarean deliveries ranged between 15 and 41 years with an average of $29.01 \pm 5.861$ years. The majority age group was 20 - 35 years old (Table 1). These data are similar to 


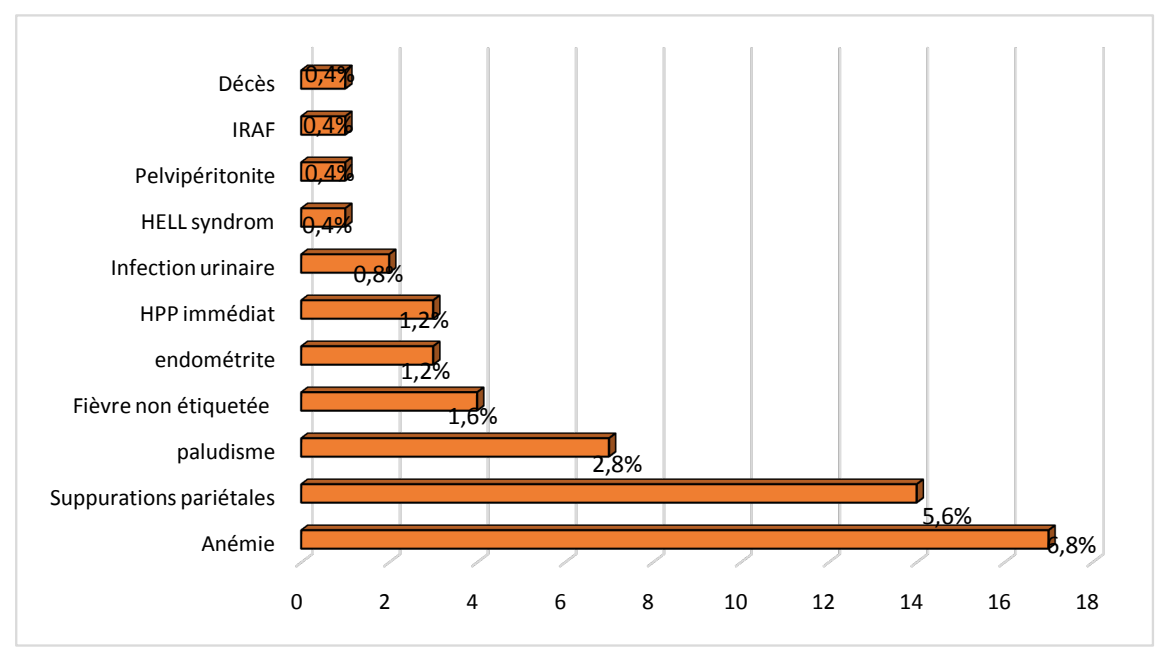

Figure 2. Summary of post-operative complications.

those reported by Togora et al.; Diawara et al. in Mali [10] [11] and corroborate the optimal range for procreation.

The caesarean section rate in our series was $29.06 \%$ of deliveries. This rate is close to that of Trabelsi et al. (28.71\%) in Tunisia in 2005; higher than the WHO recommended rate of $5 \%-15 \%$, also above the $18.64 \%$ and $23.73 \%$ reported by Kemfang et al. respectively at Yaoundé Central Hospital (HCY) and Yaoundé General Hospital (HGY) in 2012, as well as the 17.9\% found by Mbongo et al. in Brazzaville [2] [4] [12] [13].

The laquintinie hospital is located at the central level (2nd category) of the health pyramid of Cameroon with a buffer mission between the district hospitals and the 1st category hospitals. As such it receives all emergencies of peripheral hospitals, which explains this rate above the WHO standards. In the illustration, the rate of $46.8 \%$ of pregnant referred in our series. In addition, the objective of a reference structure is qualitative and not accounting. For this purpose, the WHO standard seems more indicative than operational here.

The city of Douala which hosts the Laquintinie Hospital is demographically first in Cameroon with a gross domestic product (G.D.P) of 70\%. This data, in our opinion, explains the difference with the studies of Kemfang et al., however, falling within the same national area and the same health stratum.

In terms of communication, $28.4 \%$ (71 cases) of women who underwent caesarean deliveries were informed by the operator of the surgical procedure and $27.1 \%$ (69 cases) were notified of the indication for surgery. These results converge with those of Some Der et al. at CHU (Centre Hospitalier et Universitaire) in Bobo-Dioulasso in 2005, which revealed that $38.5 \%$ of patients were informed about the procedure and diagnosis [9]; But inferior to those of Muthukumarappan et al. who reported that $43 \%$ of the patients had received caesarean section counselling [14]. The painfulness of the work as reported in the state of the place seems to justify this failure. Because the gynaecologist on duty has to manage gynaecological and obstetric emergencies but also to give advice and conduct in 
the units while ensuring continuity of care.

In our series, $95.6 \%$ of caesareans were performed more than 1 hour after indication (Figure 2). The average length of the turnaround time was 214 minutes, about $3 \mathrm{~h} 56$ minutes. These results are superimposable to those of Mbongo et al. who reported an average delay of 240 minutes [15], but well above the $35 \mathrm{~min}$ utes of the Dembele et al. (Mali) study, to the $1 \mathrm{~h} 12$ minutes found by Some Der et al. at the CHU Bobo-Dioulasso (Burkina Faso) in 2005 [3] [9] they are also well above the norms and recommendations of American and Canadian learned societies (RCOG, American College of Obstetricians and Gynaecologists, National consensus conference in Canada and United kingdom confidential inquiry) that advocate foetal extraction within 30 minutes after decision in emergency caesarean operation [16] [17].

When analysing our data, we put to the credit of these great differences the endogenous and exogenous causes.

The endogenous causes are of procedural nature: the long queues in front of the cash office, especially at night because of the small number of cashiers on duty; anything that affects the assignment of kits and other consumables. The presence of a single anaesthetist staff for all surgical emergencies of the hospital further extends these care times during calls.

Exogenous causes are often dependent on the financial difficulties of the families of the respondents. Cameroon does not have a community health insurance system. The occupational profile (mostly unemployed) of our caesarean deliveries corroborates our explanations. Because $33 \%$ of our series was without income and $26 \%$ had uncertain income because working in the informal sector. As the surgical act is sociologically feared in our communities, it is legion that the family consults for this purpose before any passage in the operating room. These difficulties are also reported by Some Der et al. in Burkina Faso and Mbongo et al. in Brazzaville [9] [15].

The operative kit was present in $98 \%$ of cases and the operating theatre was available in a proportion of $93 \%$ of cases.

The effective presence of the surgical team associated with the quasi availability of the kit and the operating theatre constitutes pertinent points to elucidate considering the great delay of action and suggests a demotivation of the staff due to the work overload. That which in our opinion is the whole problem to solve are the long delays of action. Correcting the queues, favouring access to consumables through a non-financial emergency procedure certainly does not improve this variable without a survey of satisfaction of the practitioners and the reinforcement of the human resources in order to reduce the load of the work.

Mechanical dystocia (21.2\%), fresh uterine scar (16.4\%) and acute foetal distress (AFD) (14\%) made up the 3 major groups of indications (Table 3). These findings corroborate the data of Dembele and Breda [3] [18]. The first indication of our study is similar to that reported by Diarra et al. in Mali in 2005 [19]. These results contrast with the studies of Aziz et al. in Pakistan and Bangalet et al. in India where the uterine scar was the first indication [20] [21]. The presence 
of the fresh uterine scar as a second indication (16.4\%) attests to the caution of the obstetricians in our study given the high risk of uterine rupture in this indication and motivated by the absence of a tocograph in our department. This is also the second indication reported by Tonyi et al. in Limbé and Buea in Cameroon [22]. The absence of a suitable technical platform for the definite diagnosis of AFD with the measurement of scalp $\mathrm{pH}$ forces obstetricians to make this diagnosis solely on the basis of variation of the foetal heartbeat and/or the abnormal colour of the amniotic fluid, justifying the presence of the AFD as 3rd indication of caesarean section in our study.

Emergency caesarean section was $91.2 \%$ (Table 4) These results are similar to those of Nana et al. $90.2 \%$ and $86.7 \%$ in two hospitals in the far north of Cameroon [23]. This is because all references were in an emergency context.

All caesareans were performed by the gynaecologist-obstetricians. In contrast to the study of Kemfang et al. in 2012 that revealed that caesareans were performed in $71 \%$ of cases at the Central Hospital Yaounde (CHY) by residents in gynaecology [1]. Unlike the obstetrics and gynaecology department of Laquintinie hospital which does not count any resident.

Intraoperative complications accounted for $3.2 \%$ of the total study population (1.2\% related to anaesthesia with cardiopulmonary arrest) (Table 5). These findings are superior to those of Diarra et al. in Bamako who found 0\% [19].

Of the 250 new-borns registered, 8 (3.1\%) were stillbirths, $26(10.4 \%)$ were in a state of apparent death and 7 died in neonatology. At discharge from the hospital, 235 new-borns were alive, i.e. a total of 15 (6\%) neonatal deaths (Table 6).

Our neonatal morbidity rate of $10.4 \%$ appears to be better despite the delays in care mentioned above and thus confirms the quality of the neonatal care received. Because our rate is lower than that of Togora in Bamako 21.7\% [10] also lower than those of Tanyi et al. in Limbe and Buea in Cameroon who found 26\% of new-borns with an unsatisfactory condition [22]. Their results correlate with those of Forsah and Tebeu [24] [25].

Unlike our practice (general anaesthesia $15 \%$ ), the study by Tanyi et al. mentions the almost frequent use of general anaesthesia (G.A). General anaesthesia has been reported in the literature as being associated with unsatisfactory neonatal outcomes [26] [27] [28]. There is also a lack of intensive neonatal care, which was decried in their study.

In our study, $21.6 \%$ of caesarean sections were complicated postoperatively (Figure 2). This rate is similar to the $20.11 \%$ found by Kemfang et al. at the CHY in 2012, at $21.4 \%$ by Hager et al in Norway in 2004 and $22.7 \%$ of Mariko et al. in 2007 in Mali [1] [4] [28]. However, our results are superior to the $7.69 \%$ found at the Yaounde General Hospital (YGH) by Kemfang et al., to those of Diarra et al. in Bamako 12\% and 5.2\% found by Dembele et al. in Mali [1] [3] [19]. Infectious complications were the most frequent, representing $10.8 \%$ of the total population (Figure 2). This rate is comparable to the $9.04 \%$ reported by Kemfang et al. at HCY, $12.6 \%$ of Shrestha in Nepal and 10\% of Cissé in Senegal [1] [29] [30]. These results are in the range found in thein the Moroccan and Gui- 
nean literature $6.7 \%$ and $29.7 \%$ respectively [31] [32].

As noted by Kemfang at CHY and Mariko at Ignace Dee Hospital in Conakry, Guinea, rupture of perioperative asepsis is a determining factor in the occurrence of infectious complications. The difficulties of refuelling in running water and the recurring failures of the sterilization chain as mentioned in the inventory, are in our opinion determinants to the occurrence of these infections aided by the non-compliance with the postoperative therapeutic protocols in $17 \%$ for defaults in financial resources. Parietal suppuration $(5.6 \%)$ is at the forefront of infectious complications. This rate is not far from that found by Diarra et al. in Bamako 4\% [19]. But higher than those of Kemfang at CHY and YGH which were respectively $1.74 \%$ and $1.70 \%$.

Death was deplored immediately postoperatively in a 24 -year-old pauciparous operated for eclampsia on a scarred uterus. In our study, overall maternal mortality (per and postoperative) was $0.8 \%$ (2 deaths) (Table 6 and Figure 2) contrasting with the absence of death in the Kemfang study at CHY and YGH [1]. But this rate is lower than that of Cissé in Senegal 1.4\% and 3.45\% in the Guinean study [30] [32]. The lower rate of maternal mortality in our study compared to theirs could be explained by the size of our sample lower than theirs.

\section{Conclusion}

Caesarean section at Laquintinie hospital is twice as high as WHO standards. Nearly half of the pregnant women who underwent caesarean sections were referred emergency cases. The average time of execution of emergency caesareans was $3 \mathrm{~h} 56$ minutes far above the 30-minute norms despite the availability of the operating kit and the operating theatre. The health-care personnel are in sufficient for post-operative care; this state creates work overload and demotivation of unmotivated personnel; which explains insufficient postoperative monitoring. Hence the need to initiate a study of staff satisfaction. We equally observe significant neonatal mortality.

\section{What Is Known}

Caesarean section supplements the vaginal way, thereby ensuring maternal and foetal well-being.

Caesarean section carries a risk of maternal and foetal morbidity and mortality.

We are witnessing a surge of caesareans in the world.

What our study contributes.

It notes a significant rate of irrelevant indications.

It reveals unsafe practice and suggests corrective approaches including a staff satisfaction survey.

\section{Contributions of the Authors}

Essome: collecting manuscript writing data and co-direction of the study, Mve, 
Engbang, Boten, Essiben, Tocki, Fumane, read and edited the manuscript, Mboudou editorial supervision, manuscript correction and direction of the manuscript study.

\section{Conflicts of Interest}

The authors declare no conflict of interest.

\section{References}

[1] Kemfang, J.D., Ngassam, A., Fouogue, J.T., Metogo, J., Medou, A. and Kasia, J.M. (2015) Complications maternelles précoces de la césarienne: à propos de 460 cas dans deux hôpitaux universitaires de Yaoundé, Cameroun. The Pan African Medical Journal, 21, 265. https://doi.org/10.11604/pamj.2015.21.265.6967 http://www.panafrican-med-journal.com/content/article/21/265/full/

[2] OMS (2015) Déclaration de l'OMS sur les taux de césarienne. Genève, 8.

[3] Dembele, M. (2008) Etude Qualitative De La Césarienne Au Service De Gynécologie Obstétrique De L'hôpital De Sikasso Du 1er Janvier 2005. Thèse de médecine. Faculté de Médecine de Pharmacie et d'Odontostomatologie De l'Université de Bamako, Bamako.

[4] Mariko, S. (2008) La césarienne au Centre de santé de référence de Koutiala: indications et pronostics foeto-maternels. thèse de médecine, Mali.

[5] OMS (2016) Audit et retour d'information: Effets sur les pratiques professionnelles et les résultats en matière de soins de santé.

http://apps.who.int/rhl/effective_practice_and_organizing_care/rpcom2/fr/

[6] Ebah essongue (2015) Cameroun: Mortalité maternelle, la tragédie silencieuse-Les échos du shabba.

http://shabba237.mondoblog.org/2015/07/01/cameroun-mortalite-maternelle-la-tra gedie-silencieuse/

[7] OMS, UNFPA, Groupe de la Banque Mondiale et Division de la population des nations unies (2015) Tendances de la mortalité maternelle: 1990-2015 Estimations de l'OMS, l'UNICEF, l'UNFPA, le Groupe de la Banque mondiale et la Division de la population des Nations Unies. http://apps.who.int/iris/bitstream/10665/204113/1/WHO_RHR_15.23_fre.pdf?ua=1

[8] Cisse, C., Faye, E., De Bernis, L., Dujardin, B. and Diadhiou, F. (1998) Césariennes au Sénégal: Couverture des besoins et qualité des services. Cahiers détudes et de recherches francophones/ Santé, 8, 369-377.

[9] Some Der, A., Ouattara, S., Barro, D., Traoré, A., Bamabara, M. and Dao, B. (2010) Audit des césariennes en milieu africain. Rwanda Medical Journal, 68, 4.

[10] Togora, M. (2004) La Césarienne de Qualité au CS Réf. C V du District de Bamako de 2000-2002: A propos de 2883 cas. Thèse de médecine, Faculté de Médecine de Pharmacie et d'Odontostomatologie, Bamako.

[11] Adamou, D., Hamadoun, S., Idrissa, T., Mamadou, O., et al. (2014) Complications post césarienne et gratuité de la césarienne au Mali = cas d'un centre de santé de district. Mali Médical, 1, 40-43.

[12] Trabelsi, K., Jedidi, J., Youch, S., Louati, D., Amouri, H., Gargouri, A., et al. (2006) Complications maternelles per-opératoires de la césarienne: à propos de 1404 cas. Journal JIM-Faculté de Médecine de Sfax, 11, 33-38.

[13] Mbongo, J.A., Butoyi, J.M., Papaudi-Ikourou, A. and Iloki, L.H. (2016) Césarienne 
en urgence au Centre Hospitalier et Universitaire de Brazzaville. Health Sciences and Diseases, 17, 1-4.

[14] Muthukumarappan, K., Rigby, C., Johanson, R. and Jones, P. (2000) Improving the Standards of Care for Women Having Caesarean Sections. Journal of Obstetrics and Gynaecology, 20, 584-588. https://doi.org/10.1080/01443610020001387

[15] Mc Kenzi, I.Z. and Cooke, I. (2002) Quel intervalle de temps raisonnable depuis la prise de décision jusqu'à l'extraction par césarienne. Conclusion à partir de 415 accouchements. British Journal of Obstetrics and Gynaecology, 109, 498-504.

[16] Royal College of Obstetrics and Gynaecologists, Clinical Effectiveness Support Unit, Royal College of Midwives (Great Britain), Royal College of Anaesthetists (Great Britain), National Childbirth Trust (Great Britain) (2001) The National Sentinel Caesarean Section Audit Report. Royal College of Obstetrics and Gynaecology, London.

[17] Coulibaly, I. (1999) La césarienne dans le service de gynécologie obstétrique de l'H.G.T de Bamako de 1992 à 1996(A propos de 3314 cas). Thèse de médecine, Bamako.

[18] Breda, Y., Eagleton, K., Cazenave, J.C. and Mianne, D. (1989) les indications actuelles de césariennes en Afrique tropicale. Médecine d Afrique noire électronique, $36,42-46$.

[19] Diarra, M. (2005) Etude de la césarienne à la maternité de l'hopitalNianankoro FOMBA de Ségou du 1er Janvier au au 31 Décembre 2005. Faculté de Médecine, de Pharmacie et d'Odonto-stomatologie, Bamako.

[20] Aziz, N., Yousfani, S. and Soomro, I. (2011) Rising Trend and Indications of Caesarean Section at the Liaquat University of Medical \& Health Sciences. Medical Channel, 17, 55-59.

[21] Bangal, V.B., Thorat, P.S., Patel, N.H. and Borawake, S.K. (2012) Caesarean Sections-Are We Doing Right? International Journal of Biomedical Research, 3, 281-284. https://doi.org/10.7439/ijbr.v3i6.540

[22] Tanyi, T.J., Atashili, J., Fon, P.N., Robert, T. and Paul, K.N. (2016) Caesarean Delivery in the Limbé and the Buea Regional Hospitals, Cameroon: Frequency, Indications and Outcomes. The Pan African Medical Journal, 24, 227.

https://doi.org/10.11604/pamj.2016.24.227.9499 http://www.panafrican-med-journal.com/content/article/24/227/full/

[23] Nana, P.N., Fomulu, J.N., Djenabou, A., Mbu, R.E., Tonye, R., Wandji, J.C., et al. (2011) Epidemio-Clinical Factors Associated with Caesarean Section in Two Referral Hospitals (Public/Faith-Based), Far-North Region, Cameroon. Clinics in Mother and Child Health, 8, 1-5. https://doi.org/10.4303/cmch/C100601

[24] Forsah, S.F. (2012) Rates, Indications and Complications of Caesarean Deliveries: The Case of Buea Regional Hospital.

[25] Tebeu, P.M., Ngassa, P., Mboudou, E., Kongnyuy, E.J., Binam, F. and Obama, M.T. (2008) Neonatal Survival Following Cesarean Delivery in Northern Cameroon. International Journal of Gynecology \& Obstetrics, 103, 259-260. https://doi.org/10.1016/j.ijgo.2008.07.001

[26] Lopes, T., Spirtos, N., Naik, R. and Monaghan, J. (2010) Bonney's Gynaecological Surgery. 11th Edition, John Wiley \& Sons, New York. https://doi.org/10.1002/9781444325225

[27] Reynolds, F. and Seed, P.T. (2005) Anaesthesia for Caesarean Section and Neonatal Acid-Base Status: A Meta-Analysis. Anaesthesia, 60, 636-653. 
https://doi.org/10.1111/j.1365-2044.2005.04223.x

[28] Häger, R.M., Daltveit, A.K., Hofoss, D., et al. (2004) Complications of Cesarean Deliveries: Rates and Risk Factors. American Journal of Obstetrics \& Gynecology, 190, 428-434. https://doi.org/10.1016/j.ajog.2003.08.037

[29] Shrestha, S., Shrestha, R., Shrestha, B. and Dongol, A. (2014) Incidence and Risk Factors of Surgical Site Infection Following Cesarean Section at Dhulikhel Hospital. Kathmandu University Medical Journal, 12, 113-116. https://doi.org/10.3126/kumj.v12i2.13656

[30] Cisse, C.T., Andriamady, C., Faye, O., Diouf, A., Bouillin, D. and Diadhou, F. (1995) Indications et pronostic de l'opération césarienne au CHU de Dakar. The Journal de Gynécologie Obstétrique et Biologie de la Reproduction, 24, 194-197.

[31] Hassan, A., Abderrahim, A., Fadila, M., Noureddine, M., Amine, H. and El Mansouri, A. (2001) Complications maternelles des césariennes: Analyse rétrospective de 3231 interventions à la maternité universitaire de Casablanca, Maroc. Cahiers d'études et de recherches francophones/ Santé, 10, 419-423.

[32] Diallo, F.B., Diallo, M.S., Bangoura, S., Diallo, A.B. and Camara, Y. (1998) Césarienne: Facteur de Réduction de Morbidité et de mortalité foeto-maternelle au Centre Hospitalier Universitaire Ignace Deen de Conakry (Guinée). Médecine d Afrique noire électronique 45, 359-364. 